\title{
Ultimate Generalization to Monotonicity for Uniform Convergence of Trigonometric Series
}

\author{
Song Ping Zhou , ${ }^{1}$ \\ Institute of Mathematics, Zhejiang Sci-Tech University, Xiasha Economic \\ Development Area, Hangzhou, Zhejiang 310018 China, and \\ Department of Mathematics, Statistics \& Computer Science, St. Francis \\ Xavier University, Antigonish, Nova Scotia, Canada B2G 2W5 \\ szhou@zjip.com \\ Ping Zhou, ${ }^{2}$ Dan Sheng $\mathrm{Yu}^{3}$ \\ Department of Mathematics, Statistics \& Computer Science, St. Francis \\ Xavier University, Antigonish, Nova Scotia, Canada B2G 2 W5 \\ pzhou@stfx.ca,dyu@stfx.ca
}

\begin{abstract}
Chaundy and Jolliffe [4] proved that if $\left\{a_{n}\right\}$ is a non-increasing (monotonic) real sequence with $\lim _{n \rightarrow \infty} a_{n}=0$, then a necessary and sufficient condition for the uniform convergence of the series $\sum_{n=1}^{\infty} a_{n} \sin n x$ is $\lim _{n \rightarrow \infty} n a_{n}=0$. We generalize (or weaken) the monotonic condition on the coefficient sequence $\left\{a_{n}\right\}$ in this classical result to the so-called mean value bounded variation condition and prove that the generalized condition cannot be weakened further. We also establish an analogue to the generalized Chaundy and Jolliffe theorem in the complex space.

2000 Mathematics Subject Classification. 42A20 42A32.

Key words and phrases. trigonometric series, uniform convergence, monotonicity, mean value bounded variation.

\footnotetext{
${ }^{1}$ Corresponding author. W. F. James Chair Professor of St. Francis Xavier University. Research also supported in part by NSF of China under grant number 10471130.

${ }^{2}$ Research suppoted by NSERC of Canada

${ }^{3}$ Research supported in part by NSERC RCD grant of St. Francis Xavier University and in part by AARMS of Canada
} 


\section{Introduction and results}

Let $\left\{a_{n}\right\}$ be a nonnegative sequence, write

$$
\sum_{n=1}^{\infty} a_{n} \sin n x
$$

as a sine series. Denote by $C_{2 \pi}$ the space of all continuous functions of period $2 \pi$ equipped with the norm

$$
\|f\|=\max _{0 \leq x \leq 2 \pi}|f(x)| .
$$

In 1916, Chaundy and Jolliffe [4] proved that if $\left\{a_{n}\right\}$ is a non-increasing (monotonic) real sequence (in symbol, $\left\{a_{n}\right\} \in \mathrm{MS}$, i.e. Monotonic Sequence) with $\lim _{n \rightarrow \infty} a_{n}=0$, then a necessary and sufficient condition for the uniform convergence of series (1) is $\lim _{n \rightarrow \infty} n a_{n}=0$. This classical result, together with other convergence results of series (1), such as $L^{1}$-convergence, $L^{p}$ convergence, and best approximation, have had many applications in analysis and attracted lots of attentions.

In order to weaken the monotonic condition on the sequence $\left\{a_{n}\right\}$ for series (1) to be uniformly convergent, several groups, led by Leindler ([8][11]), Stanojevic ([15]-[17])), Telyakovskii ([18]-[21]), S. P. Zhou ([7],[23][25]), Belov ([2]), as well as Tikhonov ([22]), etc., have been working on this problem in various ways for decades and trying to find the best way to weaken the monotonic condition of the sequence $\left\{a_{n}\right\}$ for the sine series to be uniformly convergent.

One way is to generalize the monotonic condition to the quasimonotone conditions. The classical definition for a sequence $\left\{a_{n}\right\}$ to be quasimonotone (in symbol, $\left\{a_{n}\right\} \in$ CQMS, i.e. Classical Quasi-Montone Sequence) is that if there is an $\alpha \geq 0$ such that $a_{n} / n^{\alpha}$ is decreasing for all $n>0$ (see $[14],[1],[5],[6],[13])$. The general definition for quasimonotone is the so-called $O$-regularly varying quasimonotone condition. Let $R(n)$ be an increasing sequence with $R(2 n) / R(n)$ bounded for all $n>0$. A sequence $\left\{a_{n}\right\}$ is said to be O-regularly varying quasimonotone sequence $\left(\left\{a_{n}\right\} \in\right.$ RVQMS) if for some $R(n)$ with the above properties, $a_{n} / R(n)$ is decreasing for all $n>0$. It is 
proved that the monotonic condition, $\left\{a_{n}\right\} \in \mathrm{MS}$, in the classical ChaundyJollif Theorem, can be generalized to $\left\{a_{n}\right\} \in$ CQMS, or more generally, $\left\{a_{n}\right\} \in$ RVQMS. There are numerous works related to this topic, for example, see [3], [12],[15]-[24].

Although the RVQMS seems very general, it is almost impossible for one to prove that a sequence $\left\{a_{n}\right\}$ (without missing any terms) is not a RVQMS. The reason is that one has to prove that the sequence $\left\{a_{n} / R(n)\right\}$ is not decreasing for any $R(n)$ with $R(2 n) / R(n)$ bounded! This may also be one of the main reasons that analysts gradually lose their interests towards RVQMS.

People then move to another direction to establish a new way of generalizing the monotonic condition by using the so-called bounded variation concept. Leindler [8] first raised the rest bounded variation condition. A nonnegative sequence $\mathbf{A}=\left\{a_{n}\right\}$ with $\lim _{n \rightarrow \infty} a_{n}=0$ is said to be a rest bounded variation sequence $\left(\left\{a_{n}\right\} \in \mathrm{RBVS}\right)$ if

$$
\sum_{k=n}^{\infty}\left|a_{k}-a_{k+1}\right| \leq C(\mathbf{A}) a_{n}
$$

holds for all $n=1,2, \cdots$ and some constant $C(\mathbf{A})$ depending only upon the sequence $\mathbf{A}$. The Chaundy-Jollif Theorem is again proved to be true in [8] if we replace the monotonic condition by the RBV condition. However, Leindler himself proved that CQMS and RBVS are not comparable in [9].

Very recently, Le and Zhou [7] introduced a condition which generalizes both CQMS and RBVS. A nonnegative sequence $\mathbf{A}=\left\{a_{n}\right\}$ is said to be a group bounded variation sequence $\left(\left\{a_{n}\right\} \in \mathrm{GBVS}\right)$ if for some given $N_{0} \geq 1$,

$$
\sum_{k=n}^{2 n}\left|a_{k}-a_{k+1}\right| \leq C(\mathbf{A}) \max _{n \leq k \leq n+N_{0}} a_{k}
$$

holds for some constant $C(\mathbf{A})$ and all $n=1,2, \cdots$. The monotonic condition in the Chaundy-Jollif Theorem is then extended to $\left\{a_{n}\right\} \in$ GBVS. Later, $\mathrm{Yu}$ and Zhou [25] introduced further the non-onesided bounded variation condition. A nonnegative sequence $\mathbf{A}=\left\{a_{n}\right\}$ is said to be a non-onesided 
bounded variation sequence $\left(\left\{a_{n}\right\} \in \mathrm{NBVS}\right)$ if

$$
\sum_{k=n}^{2 n}\left|a_{k}-a_{k+1}\right| \leq C(\mathbf{A})\left(a_{n}+a_{2 n}\right)
$$

holds for some constant $C(\mathbf{A})$ and all $n=1,2, \cdots$. Again the monotonic condition in the Chaundy-Jollif Theorem is extended further to $\left\{a_{n}\right\} \in$ NBVS.

Another recent temptation of generalization is to the so-called almost monotonic sequence. A nonnegative sequence $\mathbf{b}=\left\{\mathbf{b}_{\mathbf{n}}\right\}$ is said to be an almost monotonic sequence $\left(\left\{b_{n}\right\} \in \mathrm{AMS}\right)$ if there is a positive constant $C($ b) such that

$$
b_{k} \leq C(\mathbf{b}) b_{n} \text { for all } k \geq n .
$$

An AMS looks easy to manage. Indeed, AMS contains RVQMS $\cup$ RBVS, but it is not comparable with GBVS, NBVS or MVBVS (see[10],[11],[25] for more discussion on this). We prove in this paper that AMS is not an option to generalize the Chaundy-Jollif Theorem:

Theorem 1 There exists a sequence $\left\{b_{n}\right\} \in$ AMS with $\lim _{n \rightarrow \infty} n b_{n}=0$ such that the series $\sum_{n=1}^{\infty} b_{n} \sin n x$ is not uniformly convergent.

Our main objective of this paper is to generalize the monotonic condition in the Chaundy-Jollif Theorem to the so-called mean value bounded variation condition and prove that the generalization achieved in this paper is final.

Definition 2 A nonnegative sequence $\mathbf{A}=\left\{a_{n}\right\}_{n=0}^{\infty}$ is said to be a mean value bounded variation sequence $\left(\left\{a_{n}\right\} \in \mathrm{MVBVS}\right)$ if there is a $\lambda \geq 2$ such that

$$
\sum_{k=n}^{2 n}\left|a_{k}-a_{k+1}\right| \leq \frac{C(\mathbf{A})}{n} \sum_{k=\left[\lambda^{-1} n\right]}^{[\lambda n]} a_{k}
$$

holds for all $n=1,2, \cdots$ and some constant $C(\mathbf{A})$ depending only upon the sequence $\mathbf{A}$.

From the definition, we can see that a MVBVS can either be non-increasing almost everywhere, or non-decreasing almost everywhere, and converge to its 
limit. We show that the class of MVBVS contains all known classes of sequences mentioned earlier, except for the AMS, as following propositions:

Proposition 3 If $\mathbf{A}=\left\{a_{n}\right\} \in$ GBVS in general sense, i.e., $\left\{a_{n}\right\}$ satisfies

$$
\sum_{k=n}^{2 n}\left|a_{k}-a_{k+1}\right| \leq C(\mathbf{A}) \max _{n \leq k<n+N_{0}} a_{k}
$$

for some given $N_{0} \geq 1$, then $\left\{a_{n}\right\} \in$ MVBVS. But the reverse is not true, i.e. there are sequences in MVBVS not satisfying (2).

Proposition 4 If $\mathbf{A}=\left\{a_{n}\right\} \in$ NBVS, then $\left\{a_{n}\right\} \in$ MVBVS. But the reverse is not true, i.e. there are sequences in MVBVS which are not in NBVS.

Our first main result is that the monotonic condition in the ChaundyJollif Theorem can be weakened to $\left\{a_{n}\right\} \in$ MVBVS:

Theorem 5 If $\mathbf{A}=\left\{a_{n}\right\} \in$ MVBVS, then a necessary and sufficient condition either for the uniform convergence of series (1), or for the continuity of its sum function $f$, is that $\lim _{n \rightarrow \infty} n a_{n}=0$.

We also prove that the MVBV condition cannot be weakened any further to guarantee the uniform convergence of the sine series (1), and therefore $\left\{a_{n}\right\} \in$ MVBVS is the ultimate generalization to the monotonic condition in Chaundy-Jollif Theorem:

Theorem 6 Let $\left\{M_{n}\right\}$ be a given nonnegative increasing sequence tending to infinity. Then there exists a sine series of the form (1) with $\lim _{n \rightarrow \infty} n a_{n}=$ 0 such that for any given $\lambda \geq 2$

$$
\lim _{n \rightarrow \infty} \frac{\sum_{k=n}^{2 n}\left|\Delta a_{k}\right|}{\frac{M_{n}}{n} \sum_{k=\left[\lambda^{-1} n\right]}^{[\lambda n]} a_{k}}=0,
$$

however, the series is not uniformly convergent. 
We prove the above propositions and theorems in next section and establish an analogue of Theorem 5 in the complex space in the last section. We will also investigate other important classic results in Fourier analysis under the MVBV condition in separate papers as continuations to this paper.

Finally in this section, we summary the generalization of the monotone conditions in the following two figures. Figure 1 shows the development of the generalization successively, while Figure 2 shows the relations of the different generalized classes of monotonic sequences. Here in Figure 2, for convenience, GBVS is when $N_{0}=1$ of the general class of GBVS.

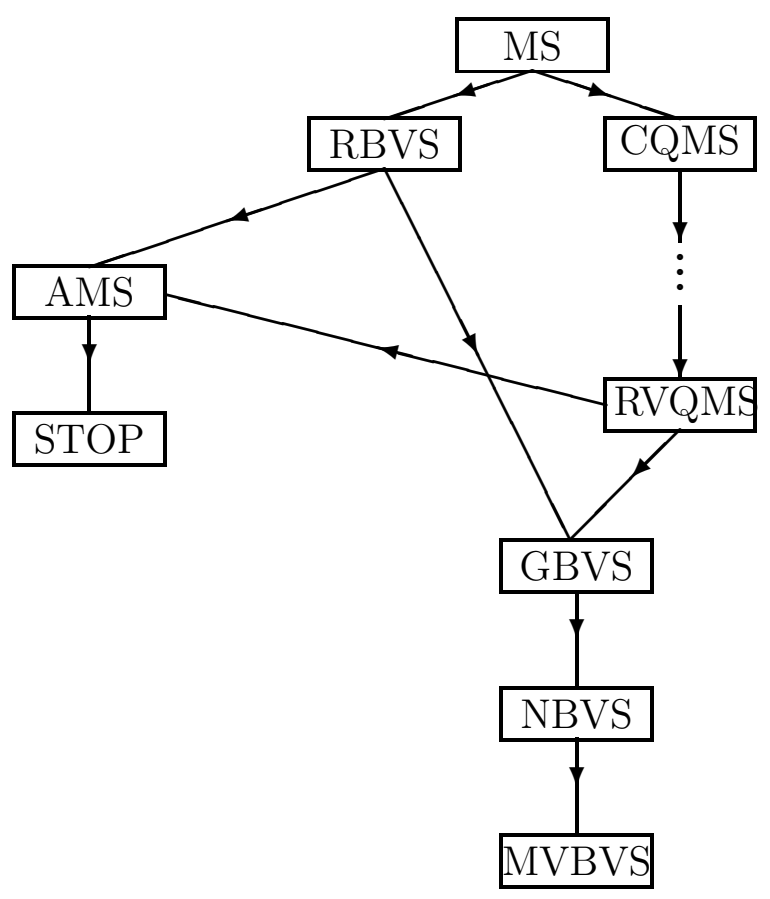

Fig.1 


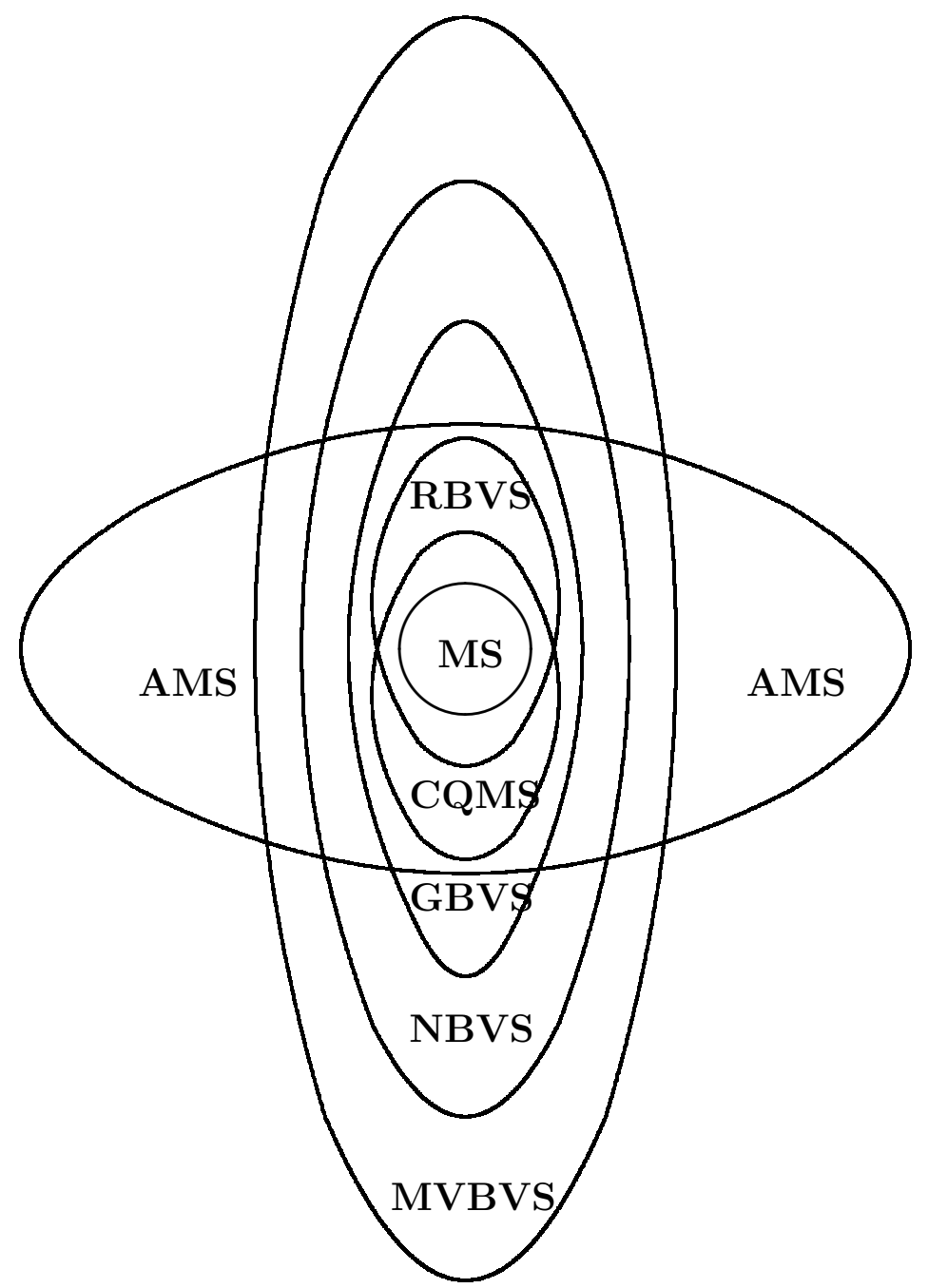

Fig.2 


\section{Proofs}

Throughout this paper, we always use $C(x)$ to denote a positive constant depending only upon $x$, where $x$ can be numbers or sequences, and use $C$ to denote an absolute positive constant. $C(x)$ or $C$ may have different values in different occurrences.

PROOF. [Proof of Theorem 1] Let $n_{1}=1, n_{2}=10, n_{j+1}=n_{j}^{2}$ for $j=2,3, \ldots$, and let

$$
b_{k}=1, \quad 1 \leq k \leq 40 .
$$

For $j \geq 2$ and $k=1,2, \ldots, n_{j}-1$, let

$$
\begin{aligned}
b_{m} & =\frac{1}{\sqrt{\log n_{j}}} \frac{1}{m}, \quad 4 k n_{j} \leq m<(4 k+2) n_{j}, \\
b_{m} & =\frac{1}{8 \sqrt{\log n_{j}}} \frac{1}{m}, \quad(4 k+2) n_{j} \leq m<4(k+1) n_{j} .
\end{aligned}
$$

Then $n b_{n} \rightarrow 0, n \rightarrow \infty$, and $b_{k} \leq 8 b_{n}$ for all $k>n$ (this means that $\left\{b_{n}\right\}$ is an almost monotonic sequence). Therefore the series $\sum_{n=1}^{\infty} b_{n} \sin n x$ is well defined. Denote by $S_{n}(x)$ the $n$th partial sum of the series, i.e.

$$
S_{n}(x):=\sum_{k=1}^{n} b_{k} \sin k x,
$$

and choose $t_{j}=\pi /\left(2 n_{j}\right)$, we have for $k=1,2, \ldots, n_{j}-1$ that

$$
\begin{aligned}
\sum_{m=4 k n_{j}}^{(4 k+2) n_{j}-1} b_{m} \sin m t_{j} & \geq \sum_{n_{j} / 2 \leq m \leq 3 n_{j} / 2} b_{4 k n_{j}+m} \sin \left(4 k n_{j}+m\right) \frac{\pi}{2 n_{j}} \\
& \geq \frac{\sqrt{2}}{2} \sum_{n_{j} / 2 \leq m \leq 3 n_{j} / 2} b_{4 k n_{j}+m} \\
& \geq \frac{\sqrt{2}}{2} \frac{1}{\sqrt{\log n_{j}}} \frac{n_{j}}{(4 k+3 / 2) n_{j}} \\
& =\sqrt{2} \frac{1}{\sqrt{\log n_{j}}} \frac{1}{8 k+3} .
\end{aligned}
$$


On the other hand,

$\left|\sum_{m=(4 k+2) n_{j}}^{4(k+1) n_{j}-1} b_{m} \sin m x\right| \leq \sum_{m=(4 k+2) n_{j}}^{4(k+1) n_{j}-1} b_{m} \leq \frac{1}{8 \sqrt{\log n_{j}}} \frac{2 n_{j}}{(4 k+2) n_{j}} \leq \frac{1}{2 \sqrt{\log n_{j}}} \frac{1}{8 k+4}$

Therefore

$$
\begin{aligned}
S_{n_{j+1}}\left(t_{j}\right)-S_{n_{j}}\left(t_{j}\right) & \geq \sum_{k=1}^{n_{j}-1}\left(\sum_{m=4 k n_{j}}^{(4 k+2) n_{j}-1} b_{m} \sin m t_{j}-\left|\sum_{m=(4 k+2) n_{j}}^{4(k+1) n_{j}-1} b_{m} \sin m x\right|\right) \\
& \geq \frac{1}{2} \frac{1}{\sqrt{\log n_{j}}} \sum_{k=1}^{n_{j}-1} \frac{1}{8 k+4} \\
& \geq \frac{C}{\sqrt{\log n_{j}}} \log n_{j} \\
& \geq C \sqrt{\log n_{j}},
\end{aligned}
$$

and the sine series is not uniformly convergent accordingly since $\sqrt{\log n_{j}} \rightarrow$ $\infty$ as $j \rightarrow \infty$.

PROOF. [Proof of Proposition 3] If $\left\{a_{n}\right\} \in$ GBVS, then for any sufficiently large $n$, we have

$$
\begin{array}{r}
\sum_{k=n}^{2 n}\left|\Delta a_{k}\right|:=\sum_{k=n}^{2 n}\left|a_{k}-a_{k+1}\right| \leq C(\mathbf{A}) \max _{n \leq k<n+N_{0}} a_{k}=: C(\mathbf{A}) a_{k_{n}}, \\
n \leq k_{n}<n+N_{0} \leq 2 n .
\end{array}
$$

For any $k_{n} / 2 \leq j \leq k_{n}$

$$
a_{k_{n}}=\sum_{i=k_{n}}^{2 j} \Delta a_{i}+a_{2 j+1} \leq \sum_{i=j}^{2 j}\left|\Delta a_{i}\right|+a_{2 j+1} \leq C(\mathbf{A}) a_{k_{j}}+a_{2 j+1},
$$

thus

$$
a_{k_{n}} \leq \frac{C(\mathbf{A})}{k_{n}} \sum_{k_{n} / 2 \leq j \leq k_{n}}\left(a_{k_{j}}+a_{2 j+1}\right) .
$$

Since $n \leq k_{n}<n+N_{0}, j \leq k_{j}<j+N_{0}$, and $k_{j}$ can repeat at most $N_{0}$ times for $k_{n} / 2 \leq j \leq k_{n}$, with the above estimate, we have 


$$
a_{k_{n}} \leq \frac{C(\mathbf{A}) N_{0}}{n} \sum_{j=[n / 2]}^{4 n+1} a_{j},
$$

which gives us a $\lambda=5$ such that

$$
\sum_{k=n}^{2 n}\left|\Delta a_{k}\right| \leq C(\mathbf{A}) a_{k_{n}} \leq \frac{C(\mathbf{A})}{n} \sum_{j=\left[\lambda^{-1} n\right]}^{\lambda n} a_{j} .
$$

On the other hand, let $n_{k}=2^{k}, k=0,1, \cdots$, set

$$
a_{n}= \begin{cases}0, & n_{k} \leq n<n_{k}+k, \\ b_{n}, & n_{k}+k \leq n<n_{k+1}-k, \\ 0, & n_{k+1}-k \leq n<n_{k+1},\end{cases}
$$

where $b_{1}, \cdots, b_{n_{k}-k}, b_{n_{k}+k}, b_{n_{k}+k+1}, \cdots, b_{n_{k+1}-k-1}, b_{n_{k+1}+k+1}, \cdots$ is any decreasing nonnegative sequence, we can easily check that $\left\{a_{n}\right\}$ does not satisfy (2) (not in NBVS either), but it certainly belongs to MVBVS.

The proof of Proposition 4 is similar to the proof of Proposition 3. Now we divide the proof of Theorem 5 into the following three lemmas. For a given series

$$
\sum_{k=1}^{\infty} a_{k} \sin k x=\lim _{n \rightarrow \infty} \sum_{k=1}^{n} a_{k} \sin k x
$$

we write

$$
f(x)=\sum_{k=1}^{\infty} a_{k} \sin k x
$$

for those points $x$ where the series converges and let $S_{n}(f, x)$ be the $n$th partial sum of $f$ at $x$. As the sequence $\left\{a_{n}\right\}$ under consideration in the sine series starts with $a_{1}$, we assume, without loss of generality, that $a_{0}=0$.

Lemma 7 Let $\left\{a_{n}\right\}$ be a nonnegative sequence and let $f(x)=\sum_{n=1}^{\infty} a_{n} \sin n x \in$ $C_{2 \pi}$. Then

$$
\lim _{n \rightarrow \infty}\left\|f-S_{n}(f)\right\|=0 .
$$

This lemma is a direct corollary of Lemma 3 in [7]. 
Lemma 8 Let $\left\{a_{n}\right\} \in$ MVBVS. Then either the uniform convergence of series (1), or the continuity of its sum function $f$, implies that $\lim _{n \rightarrow \infty} n a_{n}=0$.

PROOF. Either the uniform convergence of series (1), or the continuity of its sum function, implies that (by Lemma 7)

$$
\lim _{n \rightarrow \infty}\left\|S_{[\lambda n]}(f)-S_{\left[\frac{n}{2 \lambda}\right]-1}(f)\right\|=0
$$

holds for any given $\lambda \geq 2$. Since $\left\{a_{n}\right\} \in$ MVBVS, there exists a $\lambda \geq 2$ such that for any integer $n>0$,

$$
\sum_{k=n}^{2 n}\left|\Delta a_{k}\right| \leq \frac{C(\mathbf{A})}{n} \sum_{k=\left[\lambda^{-1} n\right]}^{[\lambda n]} a_{k} .
$$

So for $j=n+1, \ldots 2 n$, we have

$$
\begin{aligned}
a_{n} & \leq \sum_{k=n}^{j-1}\left|\Delta a_{k}\right|+a_{j} \\
& \leq \sum_{k=\left[\frac{j}{2}\right]}^{2\left[\frac{j}{2}\right]}\left|\Delta a_{k}\right|+a_{j} \\
& \leq \frac{C(\mathbf{A})}{n} \sum_{k=\left[\frac{j}{2 \lambda}\right]}^{[\lambda j / 2]} a_{k}+a_{j} \\
& \leq \frac{C(\mathbf{A})}{n} \sum_{k=\left[\frac{n}{2 \lambda}\right]}^{[\lambda n]} a_{k}+a_{j} .
\end{aligned}
$$

Taking the sum of the $n$ inequalities of (3) for $j$ runs from $n+1$ to $2 n$, we have

$$
n a_{n} \leq C(\mathbf{A}) \sum_{k=\left[\frac{n}{2 \lambda}\right]}^{[\lambda n]} a_{k}+\sum_{j=n+1}^{2 n} a_{j} \leq C(\mathbf{A}) \sum_{k=\left[\frac{n}{2 \lambda}\right]}^{[\lambda n]} a_{k} .
$$

Please note that $C(\mathbf{A})$ may have different values in different occurrences. Now let $t_{n}=\pi /(2 \lambda n)$. Then we have

$$
S_{[\lambda n]}\left(f, t_{n}\right)-S_{\left[\frac{n}{2 \lambda}\right]-1}\left(f, t_{n}\right) \geq C(\mathbf{A}) \sum_{k=\left[\frac{n}{2 \lambda}\right]}^{[\lambda n]} a_{k} \geq C(\mathbf{A}) n a_{n}
$$

and the required result follows. 
Lemma 9 Let $\left\{a_{n}\right\} \in$ MVBVS. Then $\lim _{n \rightarrow \infty} n a_{n}=0$ implies that $\lim _{n \rightarrow \infty}\left\|f-S_{n}(f)\right\|=0$.

Proof. We need only to show that

$$
\lim _{n \rightarrow \infty}\|I(x)\|:=\lim _{n \rightarrow \infty}\left\|\sum_{k=n}^{\infty} a_{k} \sin k x\right\|=0 .
$$

In view of $I(0)=I(\pi)=0$, we may restrict $x$ within $(0, \pi)$. From the condition, for any given $\varepsilon>0$, there is a $n_{0}>0$ such for all $n \geq n_{0}$ that $n a_{n}<\varepsilon$. Let $n \geq \lambda n_{0}$, where $\lambda \geq 2$ is the number obtained from the given sequence $\left\{a_{n}\right\} \in$ MVBVS, by the definition for MVBVS. Take $N=[1 / x]$ and set

$$
I(x)=\sum_{k=n}^{N-1} a_{k} \sin k x+\sum_{k=N}^{\infty} a_{k} \sin k x=: I_{1}(x)+I_{2}(x),
$$

where, without loss of generality, we assume that $N>n$, if $N \leq n$, the same argument as in estimating $I_{2}$ can be applied to deal with $\sum_{k=n}^{\infty} a_{k} \sin k x$ directly. Obviously,

$$
\left|I_{1}(x)\right| \leq x \sum_{k=n}^{N-1} k a_{k} \leq x(N-1) \varepsilon \leq \varepsilon .
$$

By the well-known estimate

$$
\left|D_{n}(x)\right|=\left|\sum_{k=1}^{n} \sin k x\right| \leq \frac{\pi}{x}
$$

and by Abel's transformation and that $\left\{a_{n}\right\} \in$ MVBVS,

$$
\begin{aligned}
\left|I_{2}(x)\right| & =\left|\sum_{k=N}^{\infty} a_{k} \sin k x\right| \\
& \leq \sum_{k=N}^{\infty}\left|\Delta a_{k}\right|\left|D_{k}(x)\right|+a_{N}\left|D_{N-1}(x)\right| \\
& \leq C x^{-1}\left(\sum_{k=N}^{\infty}\left|\Delta a_{k}\right|+a_{N}\right) \\
& \leq C N \sum_{k=N}^{\infty}\left|\Delta a_{k}\right|+\varepsilon .
\end{aligned}
$$


We check that

$$
\begin{aligned}
\sum_{k=N}^{\infty}\left|\Delta a_{k}\right| & =\sum_{j=0}^{\infty} \sum_{2^{j}} \mid \Delta \leq k<2^{j+1} N \\
& \leq C(\mathbf{A}) \sum_{j=0}^{\infty} \frac{1}{2^{j} N} \sum_{k=\left[\lambda^{-1} 2^{j} N\right]}^{\left[\lambda 2^{j} N\right]} a_{k} \\
& \leq C(\mathbf{A}) N^{-1} \varepsilon \sum_{j=0}^{\infty} 2^{-j} \sum_{k=\left[\lambda^{-1} 2^{j} N\right]}^{\left[\lambda 2^{j} N\right]} k^{-1} \\
& \leq C(\mathbf{A}, \lambda) N^{-1} \varepsilon \sum_{j=0}^{\infty} 2^{-j} \\
& \leq C(\mathbf{A}, \lambda) N^{-1} \varepsilon,
\end{aligned}
$$

since $\left\{a_{n}\right\} \in$ MVBVS. Combining all the above estimates, we have the required result.

PROOF. [Proof of Theorem 6] The construction is to be processed in a similar but more delicate way to the proof of Theorem 1. Without loss of generality, we can assume that $M_{1} \geq 10$, therefore $M_{j} \geq 10$ for all $j \geq 1$. Set $n_{1}=1, n_{2}=10$, and $n_{j+1}=2\left[M_{4 n_{j}}^{1 / 2}\right] n_{j}$ for $j=2,3, \ldots$ Let

$$
a_{k}=1, \quad 1 \leq k<40 .
$$

For $j \geq 2$ and $k=1,2, \cdots, 2\left[M_{4 n_{j}}^{1 / 2}\right]-1$, let

$$
\begin{aligned}
& a_{m}=\frac{1}{\sqrt{\log M_{4 n_{j}}}} \frac{1}{m}, \quad \text { if } 4 k n_{j} \leq m<(4 k+2) n_{j}, \\
& a_{m}=\frac{1}{8 \sqrt{\log M_{4 n_{j}}}} \frac{1}{m}, \quad \text { if }(4 k+2) n_{j} \leq m<4(k+1) n_{j} .
\end{aligned}
$$

Define accordingly a sine series $\sum_{m=1}^{\infty} a_{m} \sin m x$, we will show that this series is exact what required to prove Theorem 6 . For any given $n$, there exists a $j \geq 2$ and a $k, 1 \leq k \leq 2\left[M_{4 n_{j}}^{1 / 2}\right]-1$, such that $4 k n_{j} \leq n<4(k+1) n_{j}$, then $8 k n_{j} \leq 2 n<8(k+1) n_{j}$. Divide the argument into two cases. 
Case $1.1 \leq k \leq\left[M_{4 n_{j}}^{1 / 2}\right]-1$. Then

$$
2 n \leq 8\left[M_{4 n_{j}}^{1 / 2}\right] n_{j}=4 n_{j+1} .
$$

We check that

$$
\begin{aligned}
\sum_{m=n}^{2 n}\left|\Delta a_{m}\right| & \leq C \sum_{m=k}^{2(k+1)} a_{4 m n_{j}} \\
& \leq \frac{C}{\sqrt{\log M_{4 n_{j}}}} \sum_{m=k}^{2(k+1)} \frac{1}{4 m n_{j}} \\
& \leq \frac{C}{\sqrt{\log M_{4 n_{j}}}} \frac{k+3}{4 k n_{j}} \\
& \leq \frac{C}{\sqrt{\log M_{4 n_{j}}}} \frac{1}{n_{j}}
\end{aligned}
$$

At the same time,

$$
\begin{aligned}
\sum_{m=n}^{2 n} a_{m} & \geq \frac{1}{8 \sqrt{\log M_{4 n_{j}}}} \sum_{m=4(k+1) n_{j}}^{8 k n_{j}} \frac{1}{m} \\
& \geq \frac{1}{8 \sqrt{\log M_{4 n_{j}}}} \frac{(4 k-3) n_{j}}{8 k n_{j}} \\
& \geq \frac{1}{64 \sqrt{\log M_{4 n_{j}}}} .
\end{aligned}
$$

Thus, by noting that $4 n_{j} \leq 4 k n_{j} \leq n \leq(4 k+1) n_{j}, k \leq\left[M_{4 n_{j}}^{1 / 2}\right]-1$, for any $\lambda \geq 2$, with (6) and (7), we have

$$
\frac{\sum_{m=n}^{2 n}\left|\Delta a_{m}\right|}{\frac{M_{n}}{n} \sum_{m=\left[\lambda^{-1} n\right]}^{[\lambda n]} a_{m}} \leq \frac{\sum_{m=n}^{2 n}\left|\Delta a_{m}\right|}{\frac{M_{n}}{n} \sum_{m=n}^{2 n} a_{m}} \leq C \frac{k n_{j}}{M_{n} n_{j}} \leq C \frac{M_{4 n_{j}}^{1 / 2}}{M_{n}} \leq C M_{n}^{-1 / 2},
$$

and the last quantity in the above inequalities obviously tends to zero as $n \rightarrow \infty$.

Case 2. $\left[M_{4 n_{j}}^{1 / 2}\right] \leq k<2\left[M_{4 n_{j}}^{1 / 2}\right]$. Similarly, we calculate for this case that (note that $2 n \leq 16\left[M_{4 n_{j}}^{1 / 2}\right] n_{j}<8 n_{j+1}$ )

$$
\begin{aligned}
\sum_{m=n}^{2 n}\left|\Delta a_{m}\right| & \leq \frac{C}{\sqrt{\log M_{4 n_{j}}}} \sum_{m=k}^{2\left[M_{4 n_{j}}^{1 / 2}\right]-1} \frac{1}{4 m n_{j}}+\frac{C}{\sqrt{\log M_{4 n_{j+1}}}} \frac{1}{4 n_{j+1}} \\
& \leq \frac{C}{\sqrt{\log M_{4 n_{j}}}} n_{j}^{-1} .
\end{aligned}
$$


On the other hand, by noting that $[n / 2] \leq 2(k+1) n_{j} \leq 4\left[M_{4 n_{j}}^{1 / 2}\right] n_{j}$ we achieve that

$$
\sum_{m=[n / 2]}^{2 n} a_{m} \geq \frac{1}{8 \sqrt{\log M_{4 n_{j}}}} \sum_{m=4\left[M_{4 n_{j}}^{1 / 2}\right] n_{j}}^{8\left[M_{4 n_{j}}^{1 / 2}\right] n_{j}-1} \frac{1}{m} \geq \frac{C}{\sqrt{\log M_{4 n_{j}}}} .
$$

Therefore, for any $\lambda \geq 2$, it follows that

$$
\frac{\sum_{m=n}^{2 n}\left|\Delta a_{m}\right|}{\frac{M_{n}}{n} \sum_{m=\left[\lambda^{-1} n\right]}^{[\lambda n]} a_{m}} \leq \frac{\sum_{m=n}^{2 n}\left|\Delta a_{m}\right|}{\frac{M_{n}}{n} \sum_{m=[n / 2]}^{2 n} a_{m}} \leq C \frac{\left[M_{4 n_{j}}^{1 / 2}\right] n_{j}}{M_{n} n_{j}} \leq C \frac{M_{4 n_{j}}^{1 / 2}}{M_{n}} \leq C M_{n}^{-1 / 2} .
$$

Combining these two cases, in any circumstance, for given $\lambda \geq 2$ we have proved

$$
\lim _{n \rightarrow \infty} \frac{\sum_{k=n}^{2 n}\left|\Delta a_{k}\right|}{\frac{M_{n}}{n} \sum_{k=\left[\lambda^{-1} n\right]}^{[\lambda n]} a_{k}}=0 .
$$

In a similar argument to the proof of Theorem 1 , by taking $t_{j}=\pi /\left(2 n_{j}\right)$, we can prove that

$$
S_{n_{j+1}}\left(f, t_{j}\right)-S_{n_{j}}\left(f, t_{j}\right) \geq \frac{C \quad}{\sqrt{\log M_{4 n_{j}}}} \sum_{k=1}^{\left[M_{4 n_{j}}^{1 / 2}\right]} \frac{1}{8 k+4} \geq C \sqrt{\log M_{4 n_{j}}},
$$

with an observation that $n a_{n} \rightarrow 0$ as $n \rightarrow \infty$, which closely depends on $M_{n} \rightarrow \infty$ as $n \rightarrow \infty$. So we conclude that the series constructed is not uniformly convergent although $n a_{n} \rightarrow 0$ as $n \rightarrow \infty$.

\section{Results in Complex Space}

Given a trigonometric series $\sum_{k=-\infty}^{\infty} c_{k} e^{i k x}:=\lim _{n \rightarrow \infty} \sum_{k=-n}^{n} c_{k} e^{i k x}$, write

$$
f(x)=\sum_{k=-\infty}^{\infty} c_{k} e^{i k x}
$$

for those points $x$ where the series converges. Denote its $n$th partial sum $\sum_{k=-n}^{n} c_{k} e^{i k x}$ again by $S_{n}(f, x)$. Define the set

$$
K\left(\theta_{0}\right):=\left\{z:|\arg z| \leq \theta_{0}, \theta_{0} \in[0, \pi / 2)\right\} .
$$


It is of great interest to establish an analogue to the Chaundy and Jolliffe theorem in complex spaces since this will include sine and cosine series as two particular cases. Previous results concerning the generalization of ChaundyJollif Theorem to complex space can be found in [7], [23], and [25], etc. In this section we establish the following

Theorem 10 Let $\mathbf{C}=\left\{c_{n}\right\}$ be a complex sequence satisfying

$$
c_{n} \in K\left(\theta_{0}\right) a n d c_{n}+c_{-n} \in K\left(\theta_{0}\right), n=1,2, \ldots
$$

for some $\theta_{0} \in[0, \pi / 2)$. If there is a $\lambda \geq 2$ such that

$$
\sum_{k=n}^{2 n}\left|c_{k}-c_{k+1}\right| \leq \frac{C(\mathbf{C})}{n} \sum_{k=\left[\lambda^{-1} n\right]}^{[\lambda n]}\left|c_{k}\right|
$$

holds for all $n=1,2, \cdots$, then the necessary and sufficient conditions for $f \in C_{2 \pi}$ and $\lim _{n \rightarrow \infty}\left\|f-S_{n}(f)\right\|=0$ are that

$$
\lim _{n \rightarrow \infty} n c_{n}=0
$$

and

$$
\sum_{n=1}^{\infty}\left|c_{n}+c_{-n}\right|<\infty
$$

Note that the condition (8) in Theorem 10 is weaker than the analogue condition (6) of Theorem 1 in Le and Zhou [7]. The proof of Theorem 10 is the result of following four lemmas.

Lemma 11 (Xie and Zhou [23], Lemma 2). If a complex sequence $\mathbf{C}=\left\{c_{n}\right\}$ satisfies (8) for some $\theta_{0} \in[0, \pi / 2)$, then $f \in C_{2 \pi}$ implies (11).

Lemma 12 If a complex sequence $\left\{c_{n}\right\}$ satisfies (8) for some $\theta_{0} \in$ $[0, \pi / 2)$, then there is a constant $C\left(\theta_{0}\right)>0$ depending only on $\theta_{0}$, such that

$$
\operatorname{Rec}_{n} \leq\left|c_{n}\right| \leq C\left(\theta_{0}\right) \operatorname{Rec}_{n}, \quad n=0,1,2, \ldots
$$


The proof of this lemma is very straightforward.

Lemma 13 If a complex sequence $\left\{c_{n}\right\}$ satisfies (8) for some $\theta_{0} \in$ $[0, \pi / 2)$ and (9), then $\lim _{n \rightarrow \infty}\left\|f-S_{n}(f)\right\|=0$ implies (10).

PROOF. As

$$
\begin{aligned}
S_{[\lambda n]}(f, x)-S_{\left[\frac{n}{2 \lambda}\right]-1}(f, x) & =\sum_{k=\left[\frac{n}{2 \lambda}\right]}^{[\lambda n]}\left(c_{k} e^{i k x}+c_{-k} e^{-k x}\right) \\
& =\sum_{k=\left[\frac{n}{2 \lambda}\right]}^{[\lambda n]} c_{k}\left(e^{i k x}-e^{-i k x}\right)+\sum_{k=\left[\frac{n}{2 \lambda}\right]}^{[\lambda n]}\left(c_{k}+c_{-k}\right) e^{-i k x},
\end{aligned}
$$

so

$$
\left|\sum_{k=\left[\frac{n}{2 \lambda}\right]}^{[\lambda n]} c_{k}\left(e^{i k x}-e^{-i k x}\right)\right| \leq\left\|S_{[\lambda n]}(f)-S_{\left[\frac{n}{2 \lambda}\right]-1}(f)\right\|+\sum_{k=\left[\frac{n}{2 \lambda}\right]}^{[\lambda n]}\left|c_{k}+c_{-k}\right| .
$$

On the other hand, if we let $x=x_{0}=\pi /(2 \lambda n)$,

$$
\begin{aligned}
\left|\sum_{k=\left[\frac{n}{2 \lambda}\right]}^{[\lambda n]} c_{k}\left(e^{i k x_{0}}-e^{-i k x_{0}}\right)\right| & \geq\left|\sum_{k=\left[\frac{n}{2 \lambda}\right]}^{[\lambda n]} \operatorname{Rec} c_{k}\left(e^{i k x_{0}}-e^{-i k x_{0}}\right)\right| \\
& =2 \sum_{k=\left[\frac{n}{2 \lambda}\right]}^{[\lambda n]} \operatorname{Rec} c_{k} \sin k x_{0} \\
& \geq C \sum_{k=\left[\frac{n}{2 \lambda}\right]}^{[\lambda n]} \operatorname{Rec} c_{k} .
\end{aligned}
$$

Now by Lemma 12 and a similar calculation to (3) in the proof of Lemma 8 , for $j=n+1, \ldots, 2 n$,

$$
\left|c_{n}\right| \leq \frac{C(\mathbf{C})}{n} \sum_{k=\left[\frac{n}{2 \lambda}\right]}^{[\lambda n]}\left|c_{k}\right|+\left|c_{j}\right| \leq C\left(\mathbf{C}, \lambda, \theta_{0}\right)\left(\frac{1}{n} \sum_{k=\left[\frac{n}{2 \lambda}\right]}^{[\lambda n]} R e c_{k}+R e c_{j}\right),
$$

and with (13), (12), and a similar calculation to (4) in the proof of Lemma 
8,

$$
\begin{aligned}
n\left|c_{n}\right| & \leq C\left(\mathbf{C}, \lambda, \theta_{0}\right)\left(\sum_{k=\left[\frac{n}{2 \lambda}\right]}^{[\lambda n]} R e c_{k}+\sum_{j=n+1}^{2 n} R e c_{j}\right) \\
& \leq C\left(\mathbf{C}, \lambda, \theta_{0}\right)\left|\sum_{k=\left[\frac{n}{2 \lambda}\right]}^{[\lambda n]} c_{k}\left(e^{i k x_{0}}-e^{-i k x_{0}}\right)\right| \\
& \leq C\left(\mathbf{C}, \lambda, \theta_{0}\right)\left(\left\|S_{[\lambda n]}(f)-S_{\left[\frac{n}{2 \lambda}\right]-1}(f)\right\|+\sum_{k=\left[\frac{n}{2 \lambda}\right]}^{[\lambda n]}\left|c_{k}+c_{-k}\right|\right)
\end{aligned}
$$

then this and Lemma 11 imply (10) if $\lim _{n \rightarrow \infty}\left\|f-S_{n}(f)\right\|=0$.

Lemma 14 If a complex sequence $\left\{c_{n}\right\}$ satisfies the conditions (8) (for some $\left.\theta_{0} \in[0, \pi / 2)\right)$ to (11), then

$$
\lim _{n \rightarrow \infty}\left\|f-S_{n}(f)\right\|=0 .
$$

PROOF. Similar to the proof of the following identity (14) under the conditions of (10) and (11), we can easily see that the sequence $\left\{S_{n}(f, x)\right\}$ is a Cauchy sequence for each $x$ and therefore it converges at each $x$. So we only need to show that

$$
\lim _{n \rightarrow \infty}\left\|\sum_{k=n}^{\infty}\left(c_{k} e^{i k x}+c_{-k} e^{-i k x}\right)\right\|=0 .
$$

For any given $\varepsilon>0$, from the conditions of (10) and (11), there exists a $n_{0}>0$, such that for all $n \geq n_{0}$, we have

$$
n\left|c_{n}\right|<\varepsilon
$$

and

$$
\sum_{k=n}^{\infty}\left|c_{k}+c_{-k}\right|<\varepsilon
$$

For $n \geq n_{0}$, write

$$
\begin{aligned}
\sum_{k=n}^{\infty}\left(c_{k} e^{i k x}+c_{-k} e^{-i k x}\right) & =\sum_{k=n}^{\infty}\left(c_{k}+c_{-k}\right) e^{i k x}+2 i \sum_{k=n}^{\infty} c_{k} \sin k x \\
& =: I_{1}(x)+2 i I_{2}(x) .
\end{aligned}
$$


From (16), we have

$$
\left|I_{1}(x)\right|<\varepsilon .
$$

Follow the same steps in the proof of (5) for Lemma 9, and using (15) and Lemma 12 instead, we have

$$
\left|I_{2}(x)\right|<\varepsilon .
$$

This complete the proof of Lemma 14.

\section{References}

[1] R. Askey, Smoothness conditions for Fourier series with monotone coefficients, Acta Sci. Math. (Szeged), 28(1967), 169-171.

[2] A. S. Belov, On sequential estimate of best approximations and moduli of continuity by sums of trigonometric series with quasimonotone coefficients, Matem. Zametki (in Russian) 51:4(1992), 132-134.

[3] W. O. Bray, V. B. Stanojevic, On the integrability of complex trigonometric series. Proc. Amer. Math. Soc. 93 (1985), no. 1, 51-58.

[4] T. W. Chaundy and A. E. Jolliffe, The uniform convergence of a certain class of trigonometric series, Proc. London Math. Soc., 15(1916), 214216.

[5] T. O. Konovich, Estimate for the best approximation of periodic functions in the metric $L^{p}$, in: The extremal problems in the theory of functions and related problems, Institute of Mathematics, Ukrainian Academy of Science, Kiev, 2003, 83-88. (In Ukrainian).

[6] A. A. Konyushkov, Best approximation by trigonometric polynomials and Fourier coefficients, Math. Sbornik (In Russian), 44(1958), 53-84.

[7] R. J. Le and S. P. Zhou, A new condition for the uniform convergence of certain trigonometric series, Acta Math. Hungar., 108(2005), 161-169.

[8] L. Leindler, On the uniform convergence and boundedness of a certain class of sine series, Anal. Math. 27(2001), 279-285. 
[9] L. Leindler, A new class of numerical sequences and its applications to sine and cosine series, Anal. Math., 28(2002), 279-286.

[10] L. Leindler, Best approximation and Fourier coefficients, Anal. Math., $31(2005), 117-129$.

[11] L. Leindler, On the relationships of seven numerical sequences, Acta Math. Hungar., to appear.

[12] S. M. Mazhar, On strong approximation of continuous functions, Anal. Math., 29(2003), 281-287.

[13] J. R. Nurcombe, On the uniform convergence of sine series with quasimonotone coefficients, J. Math. Anal. Appl., 166(1992), 577-581.

[14] S. M. Shah, Trigonometric series with quasi-monotone coefficients, Proc. Amer. Math. Soc. 13(1962), 266-273.

[15] V. B. Stanojevic, $O$-regularly varying convergence moduli of Fourier and Fourier-Stieltjes series. Math. Ann. 279 (1987), no. 1, 103-115.

[16] V. B. Stanojevic, $L^{1}$-convergence of Fourier series with $O$-regularly varying quasimonotone coefficients, J. Approx. Theory, 60(1990), 168-173.

[17] V. B. Stanojevic, Fourier and trigonometric transforms with complex coefficients regularly varying in mean. Fourier analysis (Orono, ME, 1992), 423-432, Lecture Notes in Pure and Appl. Math., 157, Dekker, New York, 1994.

[18] S. A. Telyakovskii, On the convergence in the metric of $L$ of trigonometic series with rarely changing coefficients. (Russian) Trudy Mat. Inst. Steklov. 200 (1991), 322-326; translation in Proc. Steklov Inst. Math. 1993, no. 2 (200), 353-359

[19] S. A. Telyakovskii, Estimation of the moduli of continuity of one-variable functions in the metric of $L$ in terms of Fourier coefficients. Ukrainian Math. J. 46 (1994), no. 5, 671-678.

[20] S. A. Telyakovskii, Uniform convergence of trigonometric series with rarely changing coefficients, Math. Notes 70(2001), 553-559. 
[21] S. A. Telyakovskii, Estimates for the integral modulus of continuity of functions with rarely changing Fourier coefficients. (Russian) Mat. Sb. 193 (2002), no. 9, 93-106; translation in Sb. Math. 193 (2002), no. 9-10, $1333-1347$.

[22] S. Tikhonov, Strong approximation of Fourier series and embedding theorems, Anal. Math. 31(2005), 183-194.

[23] T. F. Xie and S. P. Zhou, The uniform convergence of certain trigonometric series, J. Math. Anal. Appl., 181(1994), 171-180.

[24] T. F. Xie and S. P. Zhou, $L^{1}$-approximation of Fourier series of complex valued functions, Proc. Royal Soc. Edinburgh, 126A(1996), 343-353.

[25] D. S. Yu and S. P. Zhou, A generalization of monotonicity condition and applications, Acta Math. Hungar., to appear. 